\title{
Comparative analysis of basic helix-loop- helix gene family among Brassica oleracea, Brassica rapa, and Brassica napus
}

Liming Miao ${ }^{1,2}$, Yingying Gao ${ }^{1,2}$, Kun Zhao ${ }^{1,2}$, Lijun Kong ${ }^{1,2}$, Shubo Yu ${ }^{1,2}$, Rongrong Li ${ }^{1,2}$, Kaiwen Liu ${ }^{1,2}$ and Xiaolin $\mathrm{Yu}^{1,2^{*}}$ (1)

\begin{abstract}
Background: The basic helix-loop-helix (bHLH) is the second largest gene family in the plant, some members play important roles in pistil development and response to drought, waterlogging, cold stress and salt stress. The bHLH gene family has been identified in many species, except for Brassica oleracea and B. napus thus far. This study aims to identify the bHLH family members in B. oleracea, B. rapa and B. napus, and elucidate the expression, duplication, phylogeny and evolution characters of them.

Result: A total of 268 bHLH genes in B. oleracea, 440 genes in B. napus, and 251 genes in B. rapa, including 21 new bHLH members, have been identified. Subsequently, the analyses of the phylogenetic trees, conserved motifs and gene structures showed that the members in the same subfamily were highly conserved. Most Ka/Ks values of homologous gene were $<1$, which indicated that these genes suffered from strong purifying selection for retention. The retention rates of BrabHLH and BolbHLH genes were 51.6 and 55.1\%, respectively. The comparative expression patterns between B. rapa and B. napus showed that they had similar expression patterns in the root and contrasting patterns in the stems, leaves, and reproductive tissues. In addition, there were 41 and 30 differential expression $\mathrm{bHLH}$ genes under the treatments of $A B A$ and JA, respectively, and the number of down regulation genes was significantly more than up regulation genes.

Conclusion: In the present study, we identified and performed the comparative genomics analysis of bHLH gene family among B. oleracea, B. rapa and B. napus, and also investigated their diversity. The expression patterns between $B$. rapa and $B$. napus shows that they have the similar expression pattern in the root and opposite patterns in the stems, leaves, and reproduction tissues. Further analysis demonstrated that some bHLH gene members may play crucial roles under the abiotic and biotic stress conditions. This is the first to report on the bHLH gene family analysis in B. oleracea and B. napus, which can offer useful information on the functional analysis of the bHLH gene in plants.
\end{abstract}

Keywords: Brassica crops, bHLH gene family, Whole genome identification, Evolutionary analysis, Expression analysis

\footnotetext{
* Correspondence: xlyu@zju.edu.cn

${ }^{1}$ Laboratory of Cell and Molecular Biology, Institute of Vegetable Science, Zhejiang University, 866 Yuhangtang Road, Zhejiang 310058, Hangzhou, China

${ }^{2}$ Key Laboratory of Horticultural Plant Growth, Development, and Quality Improvement, Ministry of Agriculture, Zhejiang Provincial Key Laboratory of Horticultural Plant Integrative Biology, Zhejiang 310058, Hangzhou, China
}

(c) The Author(s). 2020 Open Access This article is distributed under the terms of the Creative Commons Attribution 4.0 International License (http://creativecommons.org/licenses/by/4.0/), which permits unrestricted use, distribution, and reproduction in any medium, provided you give appropriate credit to the original author(s) and the source, provide a link to the Creative Commons license, and indicate if changes were made. The Creative Commons Public Domain Dedication waiver (http://creativecommons.org/publicdomain/zero/1.0/) applies to the data made available in this article, unless otherwise stated. 


\section{Background}

B. oleracea L. is an extremely important cruciferous vegetable worldwide that contains rich nutrients and has a morphologically abundant variation. B. oleracea L. generally, includes many common foods as cultivars, including cabbage, broccoli, cauliflower, kale, Brussels sprouts, collard greens, savoy and kohlrabi. Some varieties also have excellent ornamental properties. Many gene families have been identified in Brassica crops belonging to the $U$ triangle since the completed genome sequencing of many species [1]. However, the basic helix-loop-helix (bHLH) gene family has not been identified in B. oleracea and B. napus up to now. In our previous study, we found that the bHLH family genes may be related to the development of pistil in turnips. The analysis of the bHLH gene family is also intended to provide some clues to the functional research of its members.

The bHLH gene family was named from its bHLH domain. This domain is composed of 50-60 amino acids that can be divided into basic amino acids with 10-15 amino acids in the N-terminal and HLH region of approximately 40 amino acids in the C-terminal [2, 3]. The bHLH transcription factors generally function as homodimers or heterodimer and interact with the E-Box (5'-CANNTG-3'), most commonly G-Box (5'CACGTG-3'), which is the cis-element of the gene promoter region [4]. The number of bHLH family members is extremely large, which is only next to MYB transcription factors. In the previous study, 133 bHLH genes were classified into 12 subfamilies in Arabidopsis; with the determination of new bHLH genes, the family was divided into 21 subfamilies [5, 6]. A total of 162 Arabidopsis bHLH and 167 rice bHLH genes were clustered into 25 subfamilies $(\mathrm{A}-\mathrm{Y})$ [3].

The bHLH transcription factors are also involved in many developmental processes in the plant. $L A X$ (OsbHLH164) is expressed in the boundary between the shoot apical meristem and the region of new meristem formation and involved in the formation of all types of axillary meristems throughout the ontogeny of a rice plant [7]. Meanwhile, many bHLH genes respond to many types of stress, such as drought, salt, and cold stresses. OsbHLH148 and OsbHLH0O6 (RERJ1) responded to drought stress through the jasmonic acid signaling pathway [8-10]. OrbHLH2 (a bHLH gene cloned from Oryzaru fipogon Griff.) overexpression could enhance salt tolerance and osmotic stress resistance in A. thaliana transgenic plants [11]. OsbHLH1, which is independent of ABA, plays a transcriptional role in cold signal transduction [12]. In addition, some bHLH genes are also involved in plant reproductive development. Silique and septum development are restricted to the basal half, and seed set is limited to the apex in the spt-2 mutant [13]. SPT (SPATULA) and IND (INDEHISCENT) interact to mediate gynoecium and fruit development by controlling auxin distribution through cooperative binding to regulatory sequences in downstream target genes [14]. HEC2-RNAi hec1 hec3 gynoecia lack any stigmatic development and have longer styles than the wild-type one, while pin-shaped inflorescences were observed in HEC overexpression lines [15]. ETTIN is also a negative regulator of $H E C$ gene expression. $H E C$ genes are possibly involved in the auxin-mediated control of gynoecium patterning [15]. Meanwhile, some bHLH genes regulate seed development, especially the color of seed coat. A loss-of-function mutant, that is, $r c$ mutant, causes rice seed coat color to change from red to white [16]. BrTT8 regulates seed coat pigment accumulation in Brassica crops by modulating the expression of the late biosynthetic genes of flavonoid [17].

Many bHLH families have been identified in various plant species to date. Ninety five members of the bHLH superfamily genome were classified into 19 subfamilies in peach [18]. A total of $113 \mathrm{bHLH}$ transcription factors were found in strawberry [19]. bHLH genes were also identified in potato, grape, and peanut [20-22]. In Brassica spp., bHLH family was identified in $B$. rapa, which contains 230 bHLH transcription factors and is classified into 24 subfamilies [23]. However, the bHLH members in B. oleracea and B. napus have not been reported. In the present study, 268, 251, and 440 genes belonging to the bHLH family were identified in B. oleracea, B. rapa, and $B$. napus, respectively. Gene and protein sequences were evaluated and compared by analyzing the protein sequences, evolution of the gene family, chromosomal localization, and structure. Moreover, the expression profiles of bHLH family genes have been analyzed in different tissues and floral developmental stages to determine their function. These results will provide some useful clues for further studies on bHLH family in Brassica crops.

\section{Results \\ Identification and phylogenetic tree analysis of bHLH genes}

According to the BLAST results and domain verification, we obtained 268 bHLH genes in B. oleracea (Table S1), 440 genes in B. napus (Table S2, Table S3), and 251 bHLH members in B. rapa, while a previous study reported 230 identified bHLH genes in B. rapa (Table S4) [23]. The newly identified genes in $B$. rapa and their names are listed in Table S4. All bHLH genes were named based on their gene ID (Table S1, S2, S3 and S4). We constructed an unrooted cladogram with domain sequences of all bHLH genes of $B$. oleracea, B. rapa, $B$. napus and $A$. thaliana (Figure S1), and branches of the bHLH genes in $A$. thaliana and $B$. rapa were labeled in 
red and green, respectively. We also constructed NJ phylogenetic trees by using the domain sequences of bHLH genes from B. oleracea, B. rapa, and B. napus, respectively (Figure S2, Figure S3, Figure S4, Figure S5). These results will help us to classify different subfamilies of bHLH genes of the three Brassica crops.

\section{Analysis of conserved short amino acid sequence and structure}

We searched 15 conserved motifs of all bHLH members from B. oleracea, B. rapa and B. napus by using MEME, and the motifs were visualized with sequence logo plot (Figure S6). All conserved motifs were with NJ phylogenetic trees, as shown in Figures S7, Figure S8, Figure S9 and the different colors represent different motifs. Then, we divided all bHLH genes into different subfamilies according the phylogenetic trees and conserved motifs. We used three methods to determine the subfamily of bHLH genes in $B$. oleracea and $B$ napus. The first one, according to the subfamiles of bHLH genes in $A$. thaliana and $B$. rapa, the subfamilies of newly identified bHLH genes in B. oleracea, B. rapa and B. napus were classified (Figure S1). The second one, we classified the newly identified bHLH genes by the distance between the branches of their phylogenetic trees (Figures S2, Figure S3, Figure S4. Figure S5). The third one, we classified them according to the conservative motifs (Figures S7, Figure S8, Figure S9). Finally, the bHLH genes from $B$. oleracea and $B$. rapa, $B$. napus AA genome, and $B$. napus $\mathrm{CC}$ genome were classified into 25, 26, 23, and 22 subfamilies, respectively. $B$. oleracea had the same subfamilies, and B. rapa was divided into $\mathrm{Ib}(1)$ and $\mathrm{Ib}(2)$ in $B$. rapa except subfamily Ib. Subfamilies X and XIV had no members in $B$. napus AA genome, while subfamilies X, XIV, and IIIb had no members in $B$. napus CC genome. In $B$. oleracea, the largest subfamily was Ib, which had 32 members, and the smallest was orphan, which had one member. Subfamily XII was the largest one in B. rapa and B. napus, while subfamilies IVd and XIV were the smallest subfamilies in B. rapa. Subfamilies IVd and IIIf were the smallest subfamilies in the $B$. napus, which had one and six members, respectively.

For the conserved short amino acid sequence analysis, two motifs (motif 1 and motif 2 ) were highly conserved throughout all bHLH genes. Genes on adjacent evolutionary branches had highly conserved motifs in B. oleracea, B. rapa, and B. napus. Highly conserved motifs were found in the same subfamily among the three Brassica crops. For example, subfamily III $(\mathrm{d}+\mathrm{e})$ had the same conserved motifs (motif 1, 2, 4, 5, 8, and 12) in three Brassica crops. Results indicated that motifs 1 and 2 were the most conserved motifs in all bHLH genes, and the motifs in the same subfamily from different Brassica crops were highly conserved.
To determine whether the number of exons and introns in different subfamilies is conserved, we analyze the intron-exon location of all bHLH members from three Brassica crops and integrated the results according to the NJ phylogenetic trees, as shown in Figure S10, Figure S11 and Figure S12. In B. oleracea, most members in some subfamilies had the similar gene structure. For instance, most of genes in subfamilies VIIIb and $\mathrm{III}(\mathrm{d}+\mathrm{e})$ had one exon and no intron. However, the numbers of some other subfamilies were diverse. For instance, subfamily Ib was the largest subfamily. In this subfamily, 16 members had 3 exons and 2 introns, 13 of which had 2 exons and 1 intron; one gene had 1 exon and without intron, BolbHLHO89 had four exons and three introns, and BolbHLH255 have six exons and five introns. B. rapa and B. napus showed similar characters with some subfamilies conserved, while some were diverse. For the conserved subfamily $(\mathrm{III}(\mathrm{d}+\mathrm{e}))$ in different Brassica crops, the gene structures of most members were conserved. In contrast to the conservation in short amino acid sequence, the gene structures in the same subfamilies were not highly conserved.

\section{Molecular characteristics analysis and chromosomal localization of bHLH genes in B. oleracea, B. rapa, and $B$. napus}

We analyzed the molecular characteristics of bHLH genes in B. oleracea, B. rapa, and B. napus (Table S1, S2, S3 and S4). In B. oleracea, the ORF length of all bHLH genes ranged from 273 bp (BolbHLH143) to 2985 bp (BolbHLH063 and BolbHLH114), and the encoding 91 994 amino acids, respectively. The gene with the largest molecular weight was BolbHLH063 (112.881 kDa), while the minimum molecular weight of all genes was $10.253 \mathrm{kDa}$ (BolbHLH143). The theoretical isoelectric point (pI) ranged from 4.49 (BolbHLH072) to 10.26 (BolbHLH250). The pI of 174 deduced proteins were $<7$ (64.9\%), thereby indicating that these encoded proteins were acidic proteins and the rest $35.1 \%$ were basic proteins (Table S1). In B. rapa, the ORF length of all bHLH genes ranged from $255 \mathrm{bp}$ (BrabHLH067) to $3606 \mathrm{bp}$ (BrabHLH198), and the minimum to maximum molecular weight was $9.799 \mathrm{kDa}$ (BrabHLH067) to $132.383 \mathrm{kDa}$ (BrabHLH198). Among all BrabHLH deduced proteins, the pI of 156 proteins $(62.2 \%)$ were $<7$, and the pI of the other 95 proteins $(37.8 \%)$ were $>7$. In B. napus, the ORF length of all bHLH genes ranged from $243 \mathrm{bp}$ (BnabHLH020) to $4320 \mathrm{bp}$ (BnabHLHO17). The pI of 295 deduced proteins (67.0\%) were $<7$, including 141 proteins in the AA genome $(65.3 \%)$ and 154 proteins in $\mathrm{CC}$ genome $(68.8 \%)$, and the $\mathrm{pI}$ of other 145 proteins were $>7$.

In B. rapa, 247 bHLH genes were distributed on 10 chromosomes (i.e., A01-A10) (Fig. 1a). For the 21 newly 


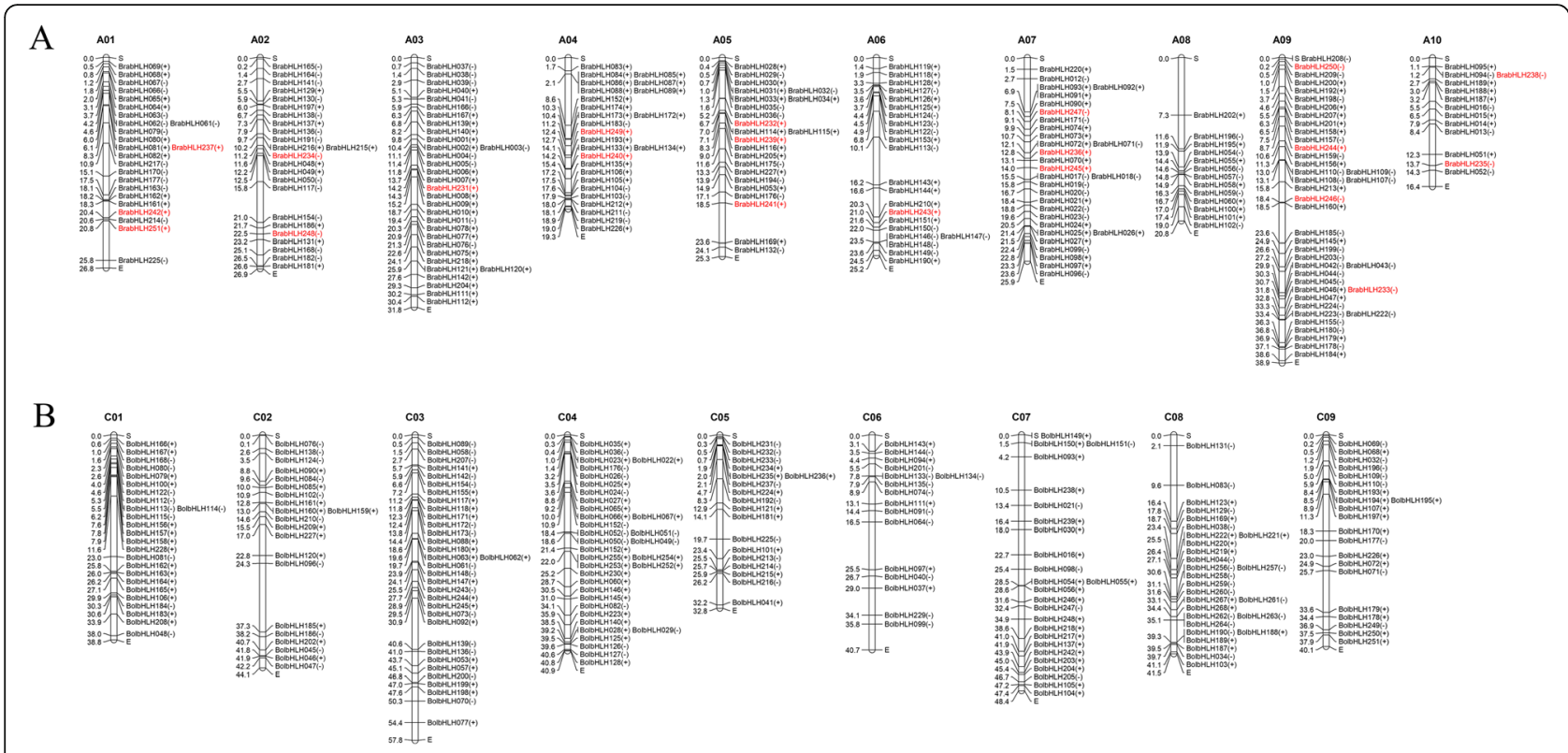

Fig. 1 Chromosome location of bHLH genes in B. rapa (a) and B. oleracea (b). The symbols + and - indicate the gene located in sense or antisense strands, respectively. The genes in red are the newly identified bHLH genes in B. rapa

identified genes, BrabHLH237, BrabHLH242, and BrabHLH251 were distributed on A01 chromosomes; BrabHLH234 and BrabHLH248 were distributed on A02; and BrabHLH231 and BrabHLH243 were distributed on A03 and A06. Two genes were located on A04 and A10, three genes were distributed on A05 and A07, and four members were mapped on A09. A total of 222 BolbHLH genes were distributed in 9 chromosomes (i.e., C01-C09) (Fig. 1b). The location of all BolbHLH genes on the chromosome can be found in Table S1. The number of bHLH genes distributed on C06 was the least, with only 16 genes, while 35 genes were distributed on C04. A total of 33 genes were distributed on the C03 chromosome, 28 genes on C08, 25 genes on C01 and C07, 21 genes on $\mathrm{C02}$ and C09, and 18 genes on C05. Finally, 46 BolbHLH genes were distributed on different scaffolds. We also mapped the bHLH genes from the $\mathrm{AA}$ and $\mathrm{CC}$ genomes of B. napus (Fig. 2). For B. napus AA genome, $190 \mathrm{bHLH}$ genes were mapped on 10 chromosomes (A01-A10). A07 had the most members (32 genes), while A08 and A10 had the minimum number of bHLH genes (11 genes). For the CC genome, 188 bHLH genes were distributed on 9 chromosomes (i.e., C01C09). C03 and C04 had the maximum number of bHLH genes (i.e., 35 genes), and the number of bHLH genes distributed on C08 was the least, with only 13 members.

\section{$K a$ and $K_{S}$ calculation of orthologous bHLH genes between $A$. thaliana and Brassica crops}

Firstly, we searched orthologous bHLH genes between A. thaliana and three Brassica crops. The $K a, K s$, and their ratio $K a / K s$ values of 205 pairs, 206 pairs and 219 pairs orthologous bHLH genes between $A$. thaliana and B. oleracea, B. rapa, B. napus were obtained, respectively (Table S5, S6, S7). The results showed that most of $K a / K s$ values of the orthologous bHLH genes between $A$. thaliana and Brassica crops were < 1 , thereby indicating that the orthologous genes suffered from strong purifying selection for retention. A total of 13, 1, and 16 pairs of genes had the $\mathrm{Ka} / \mathrm{Ks}$ value of $>1$ in $B$. oleracea, $B$. rapa and $B$. napus, which suffered from positive Darwinian selection. For B. oleracea, we linearized 211 pairs of ortholog genes distributed on the chromosomes by using the Circos program (Fig. 3). For the bHLH gene family, chromosome Chr1 had additional orthologous genes with chromosomes $\mathrm{C} 05$ and $\mathrm{C} 06$ in B. oleracea. Chr2 and C04, Chr4 and C01, Chr5 and C02, and C03 had additional orthologous genes. The orthologous bHLH genes on Chr3 were dispersed on $B$. oleracea chromosomes.

The $K s$ values of the orthologous genes can be used to calculate the divergence time between $B$. oleracea and $A$. thaliana. We first counted the distribution frequency of the $K s$ value and then calculated the divergence time on the basis of the neutral substitution rate of $1.5 \times 10^{-8}$ substitutions per site per year for Chs [24]. In the present study, the $K s$ values had a concentrated location between 0.3 and 0.4 in B. oleracea, B. rapa and B. napus (Fig. 4). This result indicated that the divergence time of the bHLH gene family between the three Brassica crops and $A$. thaliana was approximately 1.0-1.3 MYA. 


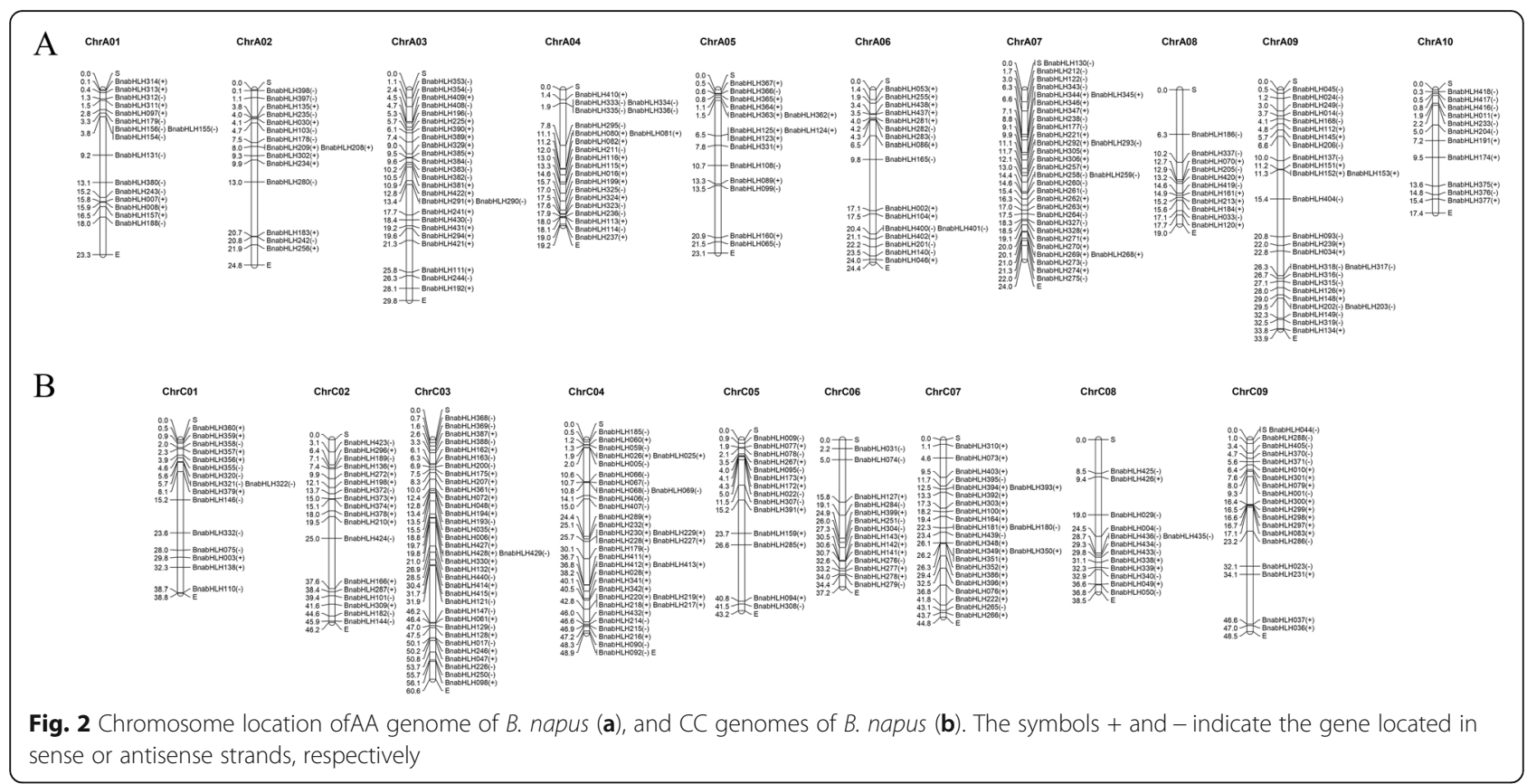

\section{Retention rates analysis of bHLH genes of three Brassica crops}

The retention rates of the identified BrabHLH and BolbHLH genes were 51.6 and $55.1 \%$, respectively (251/486 and 268/486). We calculated the retention rate of the AtbHLH orthologous bHLH, core, and random genes on different subgenomes of varying species (Fig. 5). All genes had the highest retention rate in the LF subgenome. In the LF and MF1 subgenomes of $B$. rapa, AtbHLH orthologous bHLH genes had much higher retention rate than the core and random genes, but had lower retention rate than the core genes in $B$. napus AA genome (Fig. $5 \mathrm{a}, \mathrm{c}$ ). The same trend was observed between $B$. oleracea and $B$. napus CC genome (Fig. 5b and d). In the MF2 subgenome, core genes had the highest retention rate in the three species. We also calculated the retention rates of Arabidopsis bHLH genes and determined the core and random genes in B. rapa, B. oleracea and $B$. napus (AA and $\mathrm{CC}$ genome). The retention rates of the $A t b H L H$ orthologous genes in $B$. rapa and $B$. oleracea were higher than those in B. napus (AA and $\mathrm{CC}$ genome) at 56 and $57 \%$, and higher than those of the core and random genes (Fig. 5e), respectively.

B. napus is a tetraploid crop derived from the cross between Brassica rapa and Brassica oleracea. We studied the bHLH gene loss, gain, and retention in $B$. napus after tetraploid procedure, aiming to understand whether the species had a preference for gene retention in a subgenome during the doubling process (Table 1). In theory, the number of bHLH genes in $B$. napus is the sum of bHLH genes in B. rapa and $B$. oleracea. In fact, the number was lower than the theoretical value due to the loss of genes. A total of 182 genes were lost, of which more genes were lost on the $\mathrm{CC}$ genome than in the AA genome. Some new genes were obtained in the sites without genes in $B$. rapa and $B$. oleracea genome. However, the gain of the genes was extremely rare. Only 13 genes, including 7 genes, were obtained in the MF2 sub genome. Some genes did not belong to the bHLH gene family, although they were the paralogous genes of a bHLH gene in B. rapa and B. oleracea. A total of 40 domain loss genes in $B$. napus were found in this study. Some identified genes did not have information about their distribution in the subgenomes, which we count as no hits. In this case, two no hits genes were found in B. rapa, 16 in B. oleracea, and 148 in B. napus. Hence, the most frequent occurrence of species in polyploidy is gene loss.

\section{Signal peptide and subcellular localization prediction of bHLH family of proteins}

In this study, we predicted the signal peptides of 268 , 251 and 440 bHLH proteins in B. oleracea, B. rapa, and $B$. napu, respectively. Only one and two members had signal peptides in B. oleracea and B. rapa, which were BolbHLH128 (Bol021805), BrabHLH084 (Bra014653), and BrabHLH168 (Bra029354, Figure S13). According to the $C, S$, and $Y$ values, the site near serine may be a potential signal peptide shear site. 


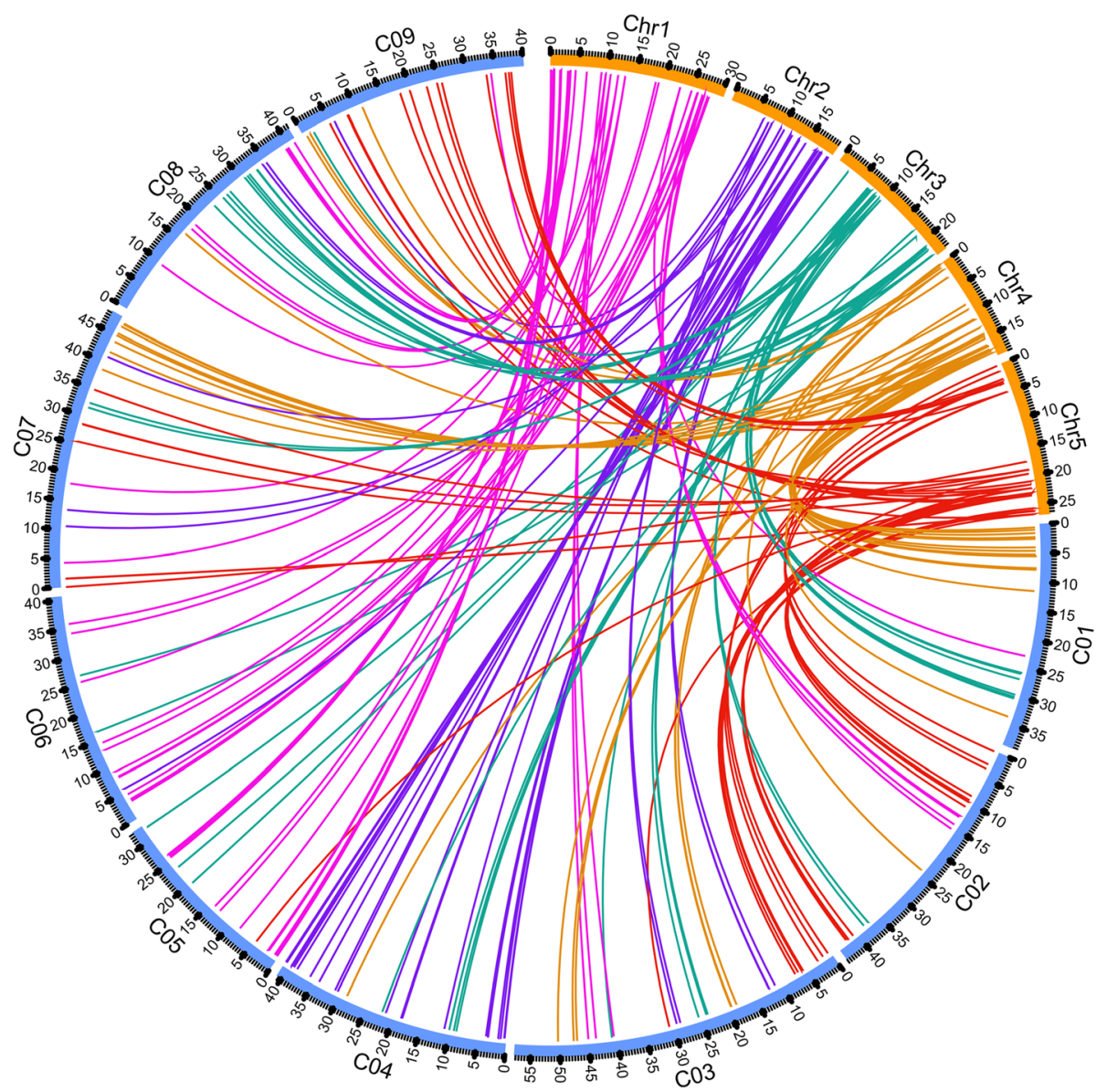

Fig. 3 Nine B. oleracea (CO1-C9) and five Arabidopsis chromosome (Chr1-Chr5) maps were based on orthologous pair positions and demonstrated highly conserved synteny. The curves of rose red, purple, green, orange, and reddish red link the bHLH gene on the Chr1, Chr2, Chr3, Chr4, and Chr5 chromosomes of A. thaliana and its orthologous genes in B. olerace, respectively
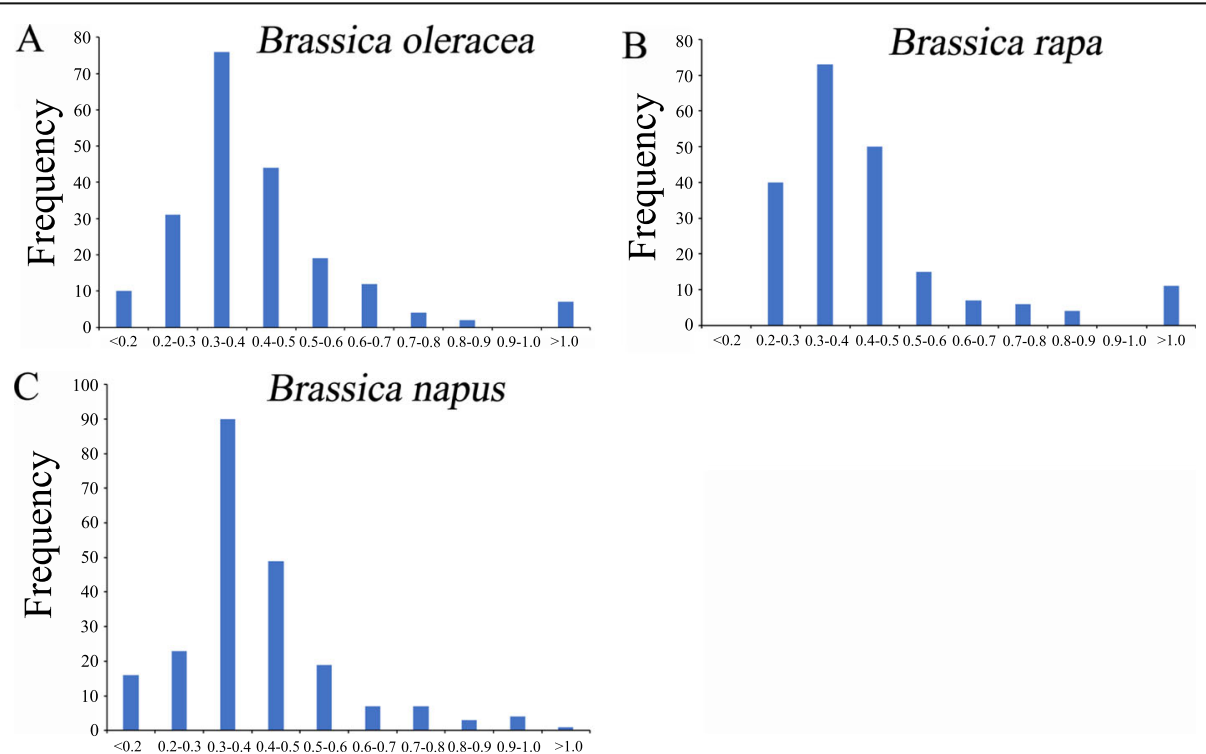

Fig. 4 Ks value distribution of orthologous bHLH genes between A. thaliana and B. oleracea (a), A. thaliana and B. rapa (b), and A. thaliana and B. napus (c). The vertical axis indicates the frequency of paired sequences. The peaks of $K s$ value appeared between 0.3 and 0.4 

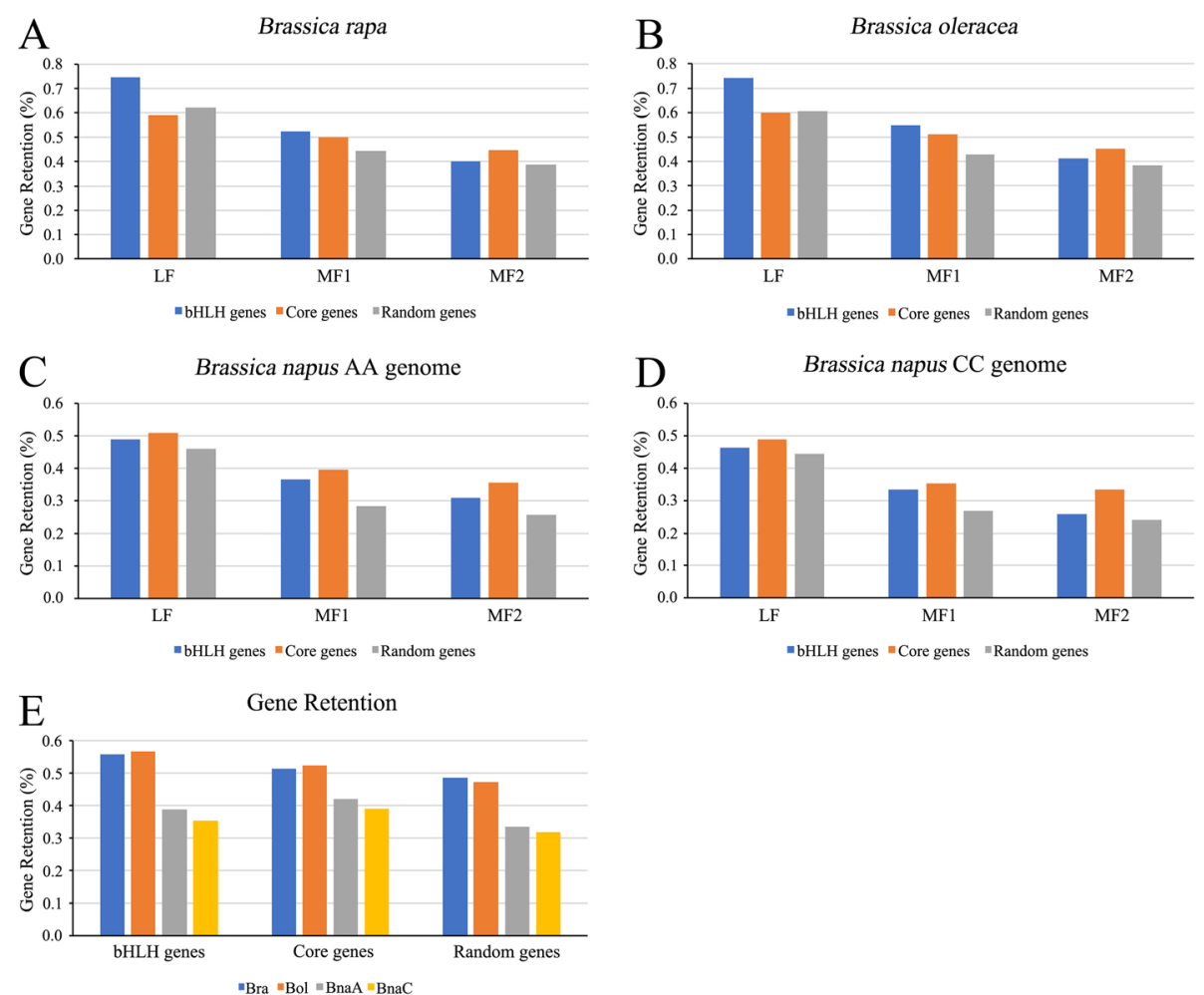

Fig. 5 Retention rates of Arabidopsis bHLH, core, and random genes in B. oleracea, $B$. rapa and B. napus. The retention rates in the different subgenomes in (a) B. rapa, (b) B. oleracea, (c) B. napus, and (d) B. napus. (e) The retention rates of Arabidopsis bHLH, core, and random genes in the whole genome of B. oleracea, B. rapa, and B. napus

To determine the subcellular localization of all bHLH proteins in the cell, we performed prediction analysis by three methods. Genes whose prediction results were inconsistent or not located in the nucleus were shown in Table S8. If the two or more of the predicted results are identical, it has been regarded as the subcellular localization of the encoded protein. With the exceptions of the genes in Table S8, all the other genes were predicted to be located in the nucleus by three methods. These results show that most of the bHLH genes were located in the nucleus, while some were located in other organelles. For example, BrabHLH037 was predicted to be located in chloroplast, cytoplasm, BrabHLH155 located in chloroplast and BolbHLH188 located in golgi

Table 1 Statistics of bHLH gene loss, gain, and retention in $B$. napus after tetraploid

\begin{tabular}{|c|c|c|c|c|c|c|c|}
\hline \multirow{2}{*}{$\begin{array}{l}\text { Gene } \\
\text { behavior }\end{array}$} & \multicolumn{2}{|l|}{ LF } & \multicolumn{2}{|l|}{ MF1 } & \multicolumn{2}{|l|}{ MF2 } & \multirow[t]{2}{*}{ Sum } \\
\hline & BnaA & BnaC & BnaA & BnaC & BnaA & BnaC & \\
\hline Loss & 39 & 42 & 30 & 34 & 16 & 21 & 182 \\
\hline Gain & 2 & 3 & 1 & 0 & 3 & 4 & 13 \\
\hline Retention & 61 & 54 & 48 & 44 & 39 & 33 & 279 \\
\hline Domain loss & 9 & 12 & 3 & 7 & 4 & 5 & 40 \\
\hline No hits & / & I & / & / & / & / & 148 \\
\hline
\end{tabular}

apparatus. There are three different prediction results of 6 genes, which are marked as not sure in Table S8. We selected 4 genes (BrabHLH245, BrabHLH051, BolbHLH207 and BnabHLH024) randomly from the Table S8 for instantaneous expression in tobacco cells, and the results showed that all of these proteins were located in the nucleus (Fig. 6). This result indicates that the predictions of subcellular localization of bHLH proteins obtained by the three methods are reliable.

Gene function annotation of bHLH proteins in $B$. oleracea We performed BLAST and GO annotation of $268 \mathrm{bHLH}$ proteins with Blast2go (Fig. 7). GO annotation (BLAST) was utilized in this study, and only one member cannot blast to the eclectic database (BolbHLH139). Finally, 267 members obtained their own function annotation. In the molecular function, most of the gene annotations were focused on DNA-binding transcription factor, DNAbinding, and protein dimerization activities. In the biological process, most annotations were concentrated in the cellular $\mathrm{N}$ compound metabolic, biosynthetic, and cellular processes. In the cellular component, most gene annotations centered on nucleus, and this result was consistent with the subcellular localization prediction (Fig. 7 and Table 1). 

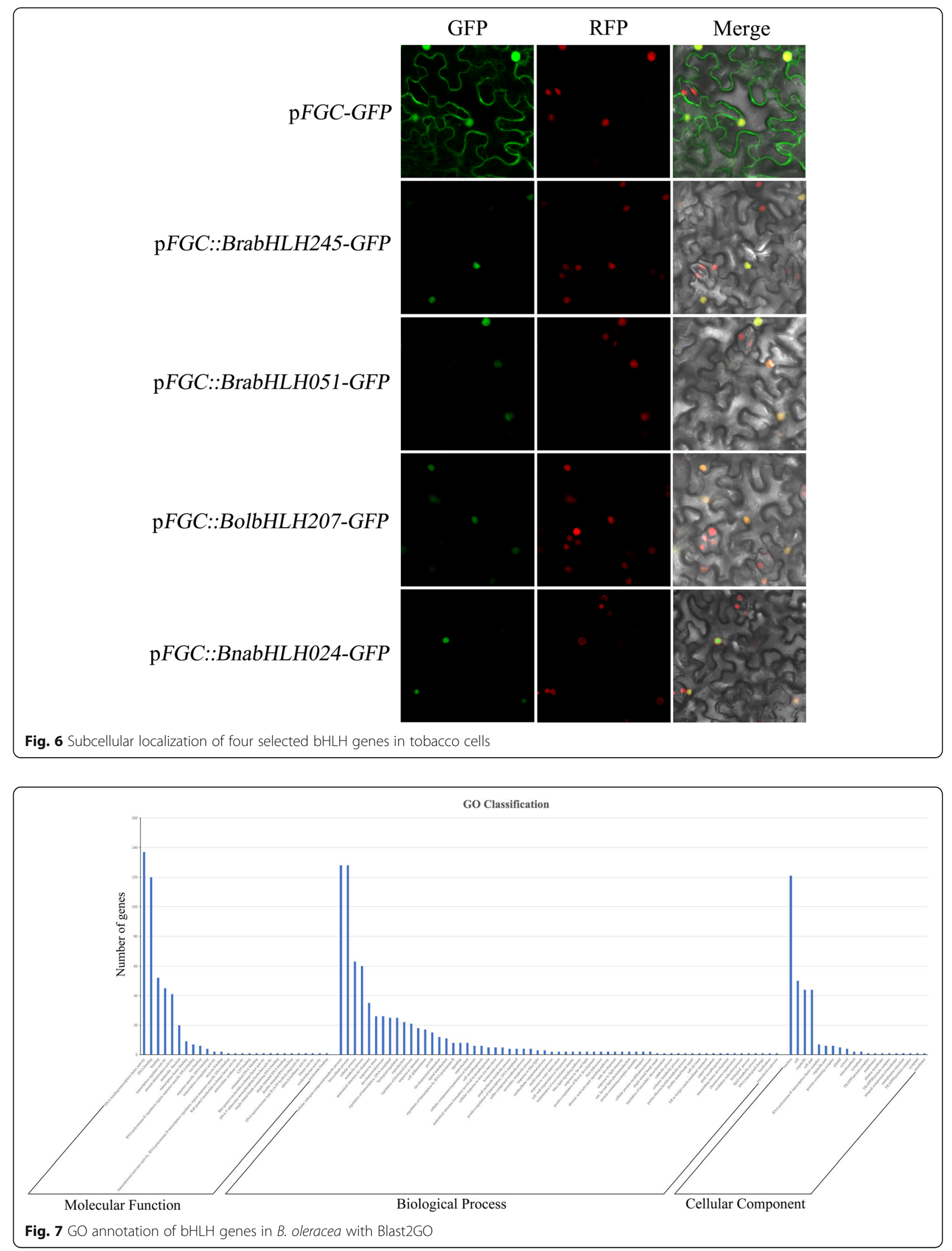
Gene expression analysis of bHLH gene family in $B$.

\section{oleracea, $B$. rapa and $B$. napus}

We analyzed the temporal and spatial expression patterns of $50 \mathrm{BrabHLH}, 50 \mathrm{BolbHLH}$ and $65 \mathrm{BnabHLH}$ genes in different tissues or organs. The original $\mathrm{Ct}$ values were showed in Table S9. All the relative expression results underwent logarithmic transformation. In $B$. oleracea, the expression patterns of the bHLH gene family were diverse. Some genes were highly expressed in vegetative tissues, while others were highly expressed in reproductive tissues (Fig. 8). For example, BolbHLHO53 had extremely high expression in the root and almost no expression in the pods and buds. By contrast, BolbHLH239 had a low expression in the root, stem, and leaf and high expression in the flower, middle buds, and small buds. The expression level decreased again with reproductive organ development.

To study whether the expression patterns of genes changed after the formation of tetraploid AACC genomes from the AA and $\mathrm{CC}$ genomes, the orthologous genes were selected for further research. The heat map showed that the expression patterns of bHLH genes in different species were diverse. However, some similarity or complementarity was observed. In $B$. rapa and $B$. napus, bHLH genes almost had no expression in the root. Nevertheless, their expression patterns in the stems, leaves, and reproductive organs were complementary. Most of genes had high expression in the stem,

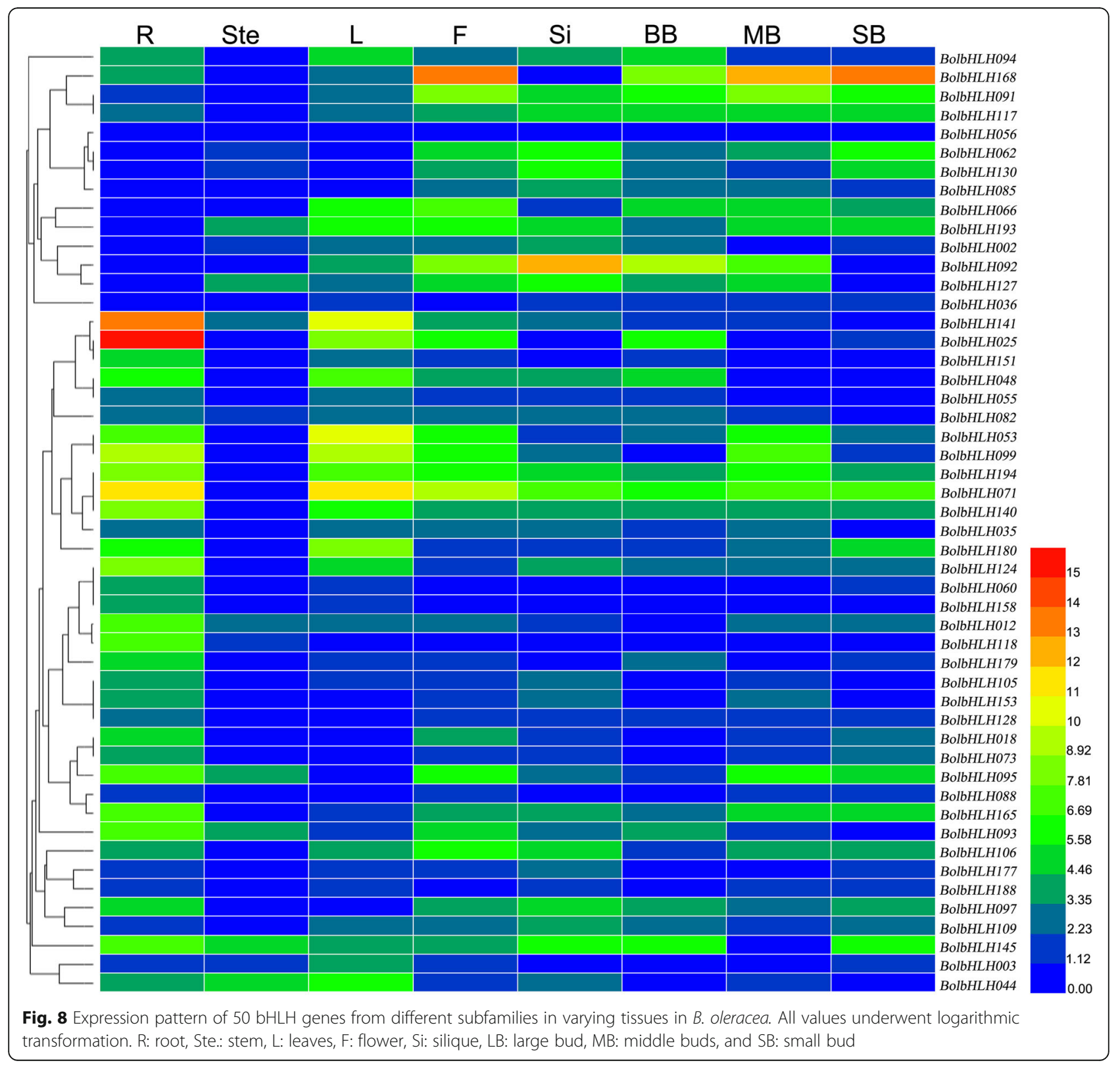


flower, silique, and floral buds and low expression in $B$. napus leaves, while $\mathrm{BrabHLH}$ genes had high expression in the leaves and low expression in the other tissues. Most of genes were highly expressed in $B$. oleracea roots, which was the opposite that in $B$. rapa and $B$. napus. Analyzing the temporal and spatial expression patterns of orthologous genes showed no significant similarity or complementarity between them (Fig. 9). For the newly identified BrabHLH genes, we analyzed the expression patterns of the two newly identified BrabHLH genes (BrabHLH238 and BrabHLH242 belonged to subfamily $\mathrm{X}$ ) and found that they had the opposite expression pattern. BrabHLH238 had no expression in the root and high expression in the stem, leaf, flower, silique, and flower buds. Meanwhile, BrabHLH242 had a slightly high expression in the root and low expression in other tissues. We also analyzed which genes were specifically expressed in certain tissues or organs, and found that 9 genes in $B$. rapa were specifically expressed in leaves, 1 gene in $B$. oleracea was specifically expressed in roots, and 1 gene in $B$. napus was specifically expressed in floral buds.

\section{Gene expression analysis of some bHLH genes under hormone treatments}

Totally, 20 BrabHLH, 20 BolbHLH and 20 BnabHLH genes have randomly been chosen to conduct gene expression analysis after ABA and JA treatments. The leaves sampled at $0 \mathrm{~h}$ after treatments were used as the control and the relative expression of others samples were calculated (Table S10). All the relative expression results underwent logarithmic transformation and visualized with a heat map (Fig. 10). For ABA treatment, the expression levels of 24 genes decreased after treatment, including $13 \mathrm{BrabHLH}$ genes, $4 \mathrm{BnabHLH}$ genes and 7 BolbHLH genes. The expression levels of 16 genes were increased in, not in B. rapa, 10 in B. napus and 6 in $B$. olerace. The expression levels increased first and then decreased of 4 BrabHLH genes, 2 BnabHLH genes and 1 BolbHLH gene. There were 13 genes of decreased expression followed by increased expression, including 2 BrabHLH genes, 3 BnabHLH genes and 8 BolbHLH genes. Among these genes, BnabHLHO30, BnabHLH363, BnabHLH310, BnabHLH323, BolbHLHO18 and BolbHLH053 have a more dramatic change in expression levels. For JA treatment, there were 17 genes of decreased expression, including 7 BrabHLH genes, 2 $B n a b H L H$ genes and 8 BolbHLH gene. The expression levels of 1 BrabHLH gene, 7 BnabHLH genes and 6 BolbHLH genes increased after treatment, BolbHLH0468 and BolbHLHO14 have large reduction; BrabHLHO37, BrabHLH020, BnabHLH134 and BrabHLHO34 have no obvious change. In addition, the expression levels of another genes were iterative processes, among them, the expression of BolbHLH062 increased rapidly at $0.5 \mathrm{~h}$ after treatment, and then decreased. Totally, the number of down regulation genes was significantly more than the number of up regulation genes.

\section{Discussion}

bHLH gene family has been identified in many species

Brassica plant is an important economic crop in the world, and B. oleracea is a widely known species. With the rapid development of bioinformatics analysis, many genome sequences of Brassica plants have been completed at present $[1,25,26]$. Several gene families have been identified in Brassica to date [27-29]. After the discovery of bHLH motif with DNA binding and dimerization, increasing bHLH protein super families have been identified in plants and animals [4]. In animals, bHLH proteins are divided into six main group (groups A to F) according to their phylogenetic relationship, motifs, and functions [6]. A total of 230 bHLH genes have been identified in B. rapa, and the expression patterns of some genes with different treatments have also been analyzed [23]. In this study, 21 new bHLH genes in $B$. rapa were identified, due to the different versions of reference genomes.

\section{The bHLH genes have been selected during the evolution} process

A. thaliana has experienced three whole genome duplication (WGD) events, as follows: a $\gamma$ event shared with most dicots and two subsequent genome duplications $(\alpha$ and $\beta$ ) shared with other members of the order Brassicales [30]. B. oleracea has a common ancestor with A. thaliana and also underwent WGD events. At approximately 1.31.7 MYA, B. oleracea experienced a whole genome triplication (WGT) event, thereby resulting in the divergence of the genome between B. oleracea and A. thaliana [31, 32]. In this study, we found that divergence time of the bHLH gene family between the three Brassica crops and A. thaliana was approximately in the range of 1.0-1.3 MYA, which is close to the result of a previous study (1.3-1.7 MYA) [33]. To reveal the selection mechanism of bHLH genes in B. oleracea, B. rapa and B. napu during evolution, we calculated the $K a / K s$ value, and most homologous gene pairs experienced purifying selection, thus indicating that these genes were strongly controlled in evolution. Only 12, 7, and 9 orthologous gene pairs had a $K a / K s$ ratio of $>1$ (Table S5, S6, S7), thereby suggesting that novel functions were likely to generate among these genes. Similar findings were observed in other gene families in plants, such as the TCS of tomato and PMEI of Brassica, of which most homolog pairs evolve through purifying selection, and a few or even no gene experiences positive selection $[34,35]$. 


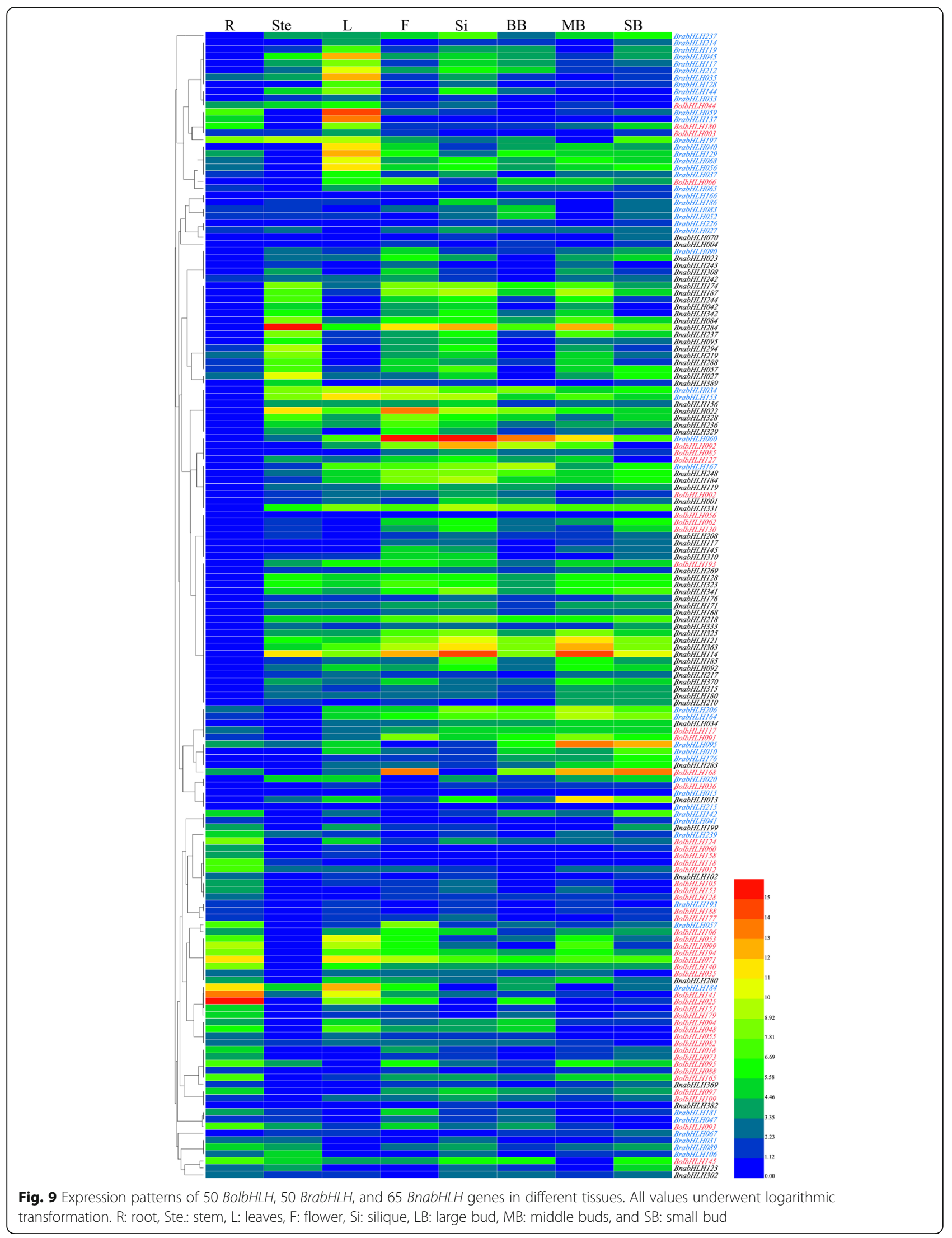




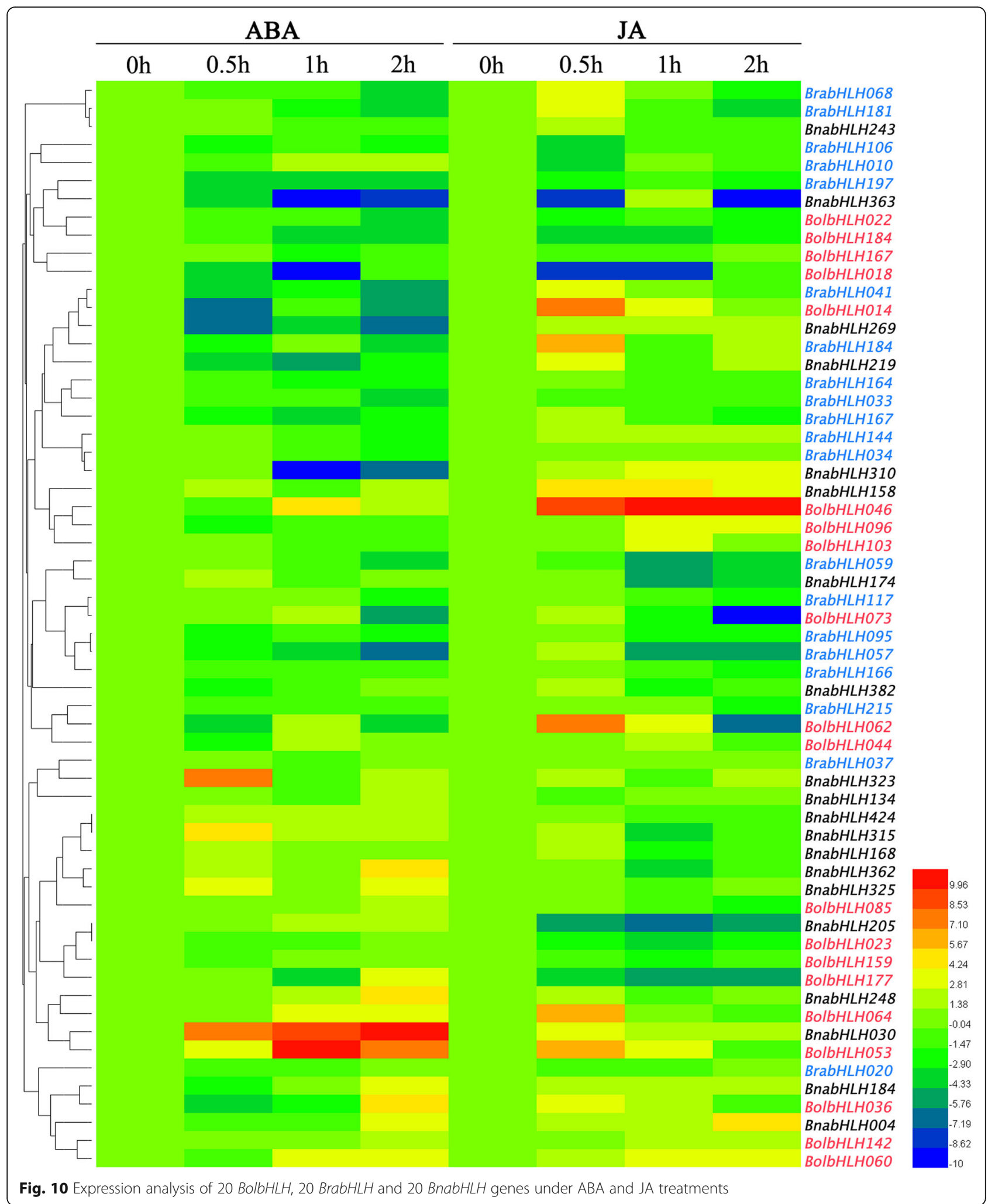

Theoretically, after WGD and WGT events, the genome size of $B$. oleracea should be threefold larger than that of $A$. thaliana. However, the size was much smaller than the theoretical value. The retention rate of $A t b H L H$ orthologous genes was $57 \%$ in B. oleracea, which was close to its retention rate $(56 \%)$ in $B$. rapa, while the 
actual retention rates in $B$. rapa and $B$. oleracea were 51.6 and $55.1 \%$. The retention rate of BolbHLH was close to that of the core and random genes and slightly higher than that of the other gene families, such as the PMEI (52\%) [35] and SDG gene families (43\%) in $B$. rapa [36]. These results of gene retentions indicated that almost half of the genes were lost after WGT event. In B. napus, the retention rates of AtbHLH orthologous, core and random genes were low. This finding suggested that about half of genes have been lost during the formation of allotetraploid procedure.

\section{The expression patterns of bHLH genes provide some clues to study their functional analysis}

bHLH gene family is an extremely large family that is involved in many regulation processes, such as stress response [37] and seed development [17, 38]. The heat map showed that the expression patterns of selected 50 BolbHLH genes were diverse. Although the functional studies on the function of bHLH genes in B. oleracea were still lacking, their gene function could be deduced via their expression patterns and also the reported gene functions of $A$. thaliana bHLH genes in the same subfamilies. INDEHISCENT, HECATE, and SPATULA are involved in the pistil development, and they belong to the VIIIb and I $(\mathrm{a}+\mathrm{b})$ subfamily, respectively [14, 15]. Our analysis of the expression patterns of BolbHLH genes belonging to the same subfamilies showed that these genes had relatively high expression in the reproductive organs, indicating their potential functions in reproductive development. After WGT, a series of events, such as chromosome rearrangement, gene loss, and epigenetic modification, often emerges [39, 40]. The remaining genes after the loss events are often associated with dosage effects or with new or subfunctionalized genes in A. thaliana [41, 42]. Homologous genes with similar expression patterns are preserved because of the dosage effect, while homologous genes with different expression patterns are retained due to new functionalization and subfunctionalization [43]. In this study, BolbHLH098/BolbHLH155, BolbHLH154/BolbHLH226, BolbHLH141/BolbHLH090, BolbHLH133/ BolbHLH159, and BolbHLH198/BolbHLH167 were homologous genes, and 4 gene pairs had similar expression pattern, except for BolbHLH141/BolbHLH090. According to the heat map, most of BolbHLH genes had similar expression patterns (Fig. 9). According to the results above, we speculated that the retention of bHLH family genes was mainly due to the dosage effect in $B$. oleracea. In our study, bHLH genes had high expression in B. oleracea leaves, while they had high expression in reproduction tissues in B. napus. The main economic organ of B. oleracea was the leaf, while that of $B$. napus was the seed. The different expression patterns may be correlated with the agronomic traits during the evolution course. In some studies, researchers have found that bHLH genes are related to plant biomass. For example, OsbHLH107 overexpression can enhance the grain size in rice, and a Vitis vinifera bHLH transcription factor $(V v C E B 1(\mathrm{opt}))$ enhances plant cell size, vegetative biomass, and reproductive yield [44, 45]. We compared the expression patterns of the specifically expressed genes between the $A$. thaliana and the three Brassica crops combined with their gene structures. The results indicated that these orthologous gene pairs have similar gene structures, while their expression patterns were diverse (Data not given). This means that their expression patterns are not related to their conserved gene structures.

The expression levels of some bHLH genes were analyzed after ABA and JA treatments. Some genes respond to ABA or JA among the selected genes in this study and the expressions of BolbHLHO64 and BnabHLHO3O changed simultaneously after ABA and JA treatments. OsbHLHOO6 (RERJ1), a jasmonic acid-responsive bHLH gene, response to drought stress and belong to $\mathrm{III}(\mathrm{a}+\mathrm{c})$ subfamily [3, 10, 23]. BrabHLH041, BolbHLH142 and $B n a b H L H 174$ of the the genes we chosen belong to III $(\mathrm{a}+\mathrm{c})$ subfamily, the expression level of BrabHLHO41, BolbHLH142 and BnabHLH174 changed after ABA and JA treatments. This indicated that the expression analysis will give some help to study their biological functions.

GO annotation of BolbHLH genes indicated their functions An important regulation pathway is that transcription factors interact with cis-acting elements to express the genes involved in stress response and developmental processes specifically. In the present study, the functional annotation of the BolbHLH genes showed that they were mainly concentrated in the DNAbinding transcription factor, DNA binding, transcription regulator, and protein dimerization activities in the molecular function section, which was consistent with the way in which the bHLH gene functions by forming homodimers or heterodimers [4]. bHLH transcription factors can regulate many genes involved in different regulatory pathways [46]. bHLH transcription factors also participate in the regulation of metabolic processes, such as the biosynthesis of alkaloids and nicotine [47]. bHLH genes are also involved in plant response to biotic and abiotic stresses. TcMYC is highly expressed in the xylem and leaves and upregulated by drought and high-salinity stresses in yew trees [48]. The CsbHLH18 of sweet orange functions in the modulation of cold tolerance by regulating the antioxidant gene [49]. Some bHLH transcription factors can also respond to salinity and Fe-deficient 
abiotic stress $[50,51]$. In the present study, the biological process of GO annotation showed that most of genes had the GO terms of cellular $\mathrm{N}$ compound, metabolic process, biosynthetic process, anatomical structure development, response to stress, regulation of transcription, DNA template, and reproduction. These enriched GO annotations were also consistent with the known functional bHLH transcription factors in some studies.

\section{Conclusion}

In this study, we identified and performed the comparative genomics analysis of bHLH gene family among $B$. oleracea, $B$. rapa and $B$. napus and also investigated their diversity. The expression patterns between $B$. rapa and $B$. napus shows that they have the similar expression pattern in the root and opposite patterns in the stems, leaves, and reproduction tissues. Further analysis demonstrated that some bHLH gene members may play a crucial role under the abiotic and biotic stress conditions. This is the first to report on the bHLH gene family in B. oleracea and B. napus. These findings can offer useful information on the functional analysis of the bHLH genes in plants.

\section{Methods}

Identification of bHLH family genes in B. oleracea, B. rapa and $B$. napus

To identify bHLH family genes in $B$. oleracea and $B$. napus, we searched the protein sequences of the 162 reported Arabidopsis bHLH from TAIR (http://www.arabidopsis.org/) and downloaded the protein sequences of 230 bHLH genes that were identified in B. rapa from the Brassica Database (http://brassicadb.org/brad/index. php) according to previous studies [5, 23, 52, 53]. We obtained the candidate genes of bHLH in $B$. oleracea and $B$. napus by analyzing the amino acid sequences of $A$. thaliana and $B$. rapa bHLH family members with BLASTP in the Brassica Database (E value $=10^{-5}$ ). We also searched all the orthologous genes of $162 \mathrm{~A}$. thaliana bHLH genes. Data of syntenic relationship between A. thaliana and different Brassica crops (like B. rapa, B. oleracea, $B$. napus and some others) are provided in Brassica database. After entering the Brassica database, select "Syntenic gene" under the menu of "Search", and then operate according to the step 1 and step 2 indicated on the page to obtain the orthologous genes of $A$. thaliana in different Brassica crops. The reference genome of B. oleracea and B. napus were provided to Brassica Database by Dr. Liu and Dr. Chalhoub, respectively [1, 541. Then, we downloaded the amino acid sequences of all candidate genes, and the Pfam database ( $\mathrm{E}$ value = 1.0, http://pfam.xfam.org/) was used to determine whether each sequence harbored the conserved domains
(HLH, PF00010.25). Genes that did not contain the known conserved domains of the gene families were excluded from further analysis. All amino acid sequences were subjected to gene ontology (GO) annotation by using Blast2GO with default parameters [55].

\section{Chromosomal localization of bHLH family genes in $B$. oleracea, $B$. rapa and $B$. napus}

We searched the start and stop locations on the B. oleracea, B. rapa and B. napus chromosomes of all bHLH family members in the Brassica database. Considering the numerous members in $B$. napus, the analysis of $B$. napus was divided into $\mathrm{AA}$ and $\mathrm{CC}$ genomes and the subsequent analyses were in the same way. Subsequently, the chromosomal localization of these members was performed using the MapChart software on the basis of the relative location of each gene on each chromosome [56].

Gene structure and phylogenetic analyses of bHLH family genes in $B$. oleracea, $B$. rapa and $B$. napus

We searched the full-length DNA and cDNA sequences of $B$. oleracea, $B$. rapa and $B$. napus bHLH genes from the National Center for Biotechnology Information, (https://www.ncbi.nlm.nih.gov/). Then, the Gene Structure Display Server database (http://gsds.cbi.pku.edu.cn/ index.php) was utilized to analyze the gene structure. The homologous sequence alignment of all bHLH genes from B. oleracea, B. rapa and B. napus was performed using ClustalW [57]. Then, the sequence alignment results were considered the basis in generating the unrooted phylogenetic tree of the $B$. oleracea, $B$. rapa and $B$. napus bHLH genes with MEGA (version 5.0) [58]. All parameters used were default parameters. Phylogenetic trees were generated with the value of the 1000 bootstrap samples by the neighbor-joining (NJ) method [58]. Then, the results of gene structure analysis were integrated with phylogenetic trees by using Photoshop CS3.

\section{Nonsynonymous substitution rate, synonymous substitution rate, and gene retention analysis} The nonsynonymous substitution rates $(K a)$ and synonymous substitution rates $(K s)$ of the orthologous bHLH gene pairs among the three Brassica crops and $A$. thaliana were calculated. First, the multiple sequence alignments of the CDS sequence pairs were performed using Clustalx [59]. Then, the values of $K a$ and $K s$ were figures out by $\mathrm{Ka} / \mathrm{Ks} \_\mathrm{Calculator}$ software with the files of sequence alignment, the method $\mathrm{YN}$ was chosen to do calculation [60]. The relationships of orthologous genes between $A$. thaliana and $B$. oleracea were also visualized using the Circos program [61]. 
The retention rates of Arabidopsis bHLH, core, and random genes and the retention rate in the different subgenomes (i.e., LF, MF1, and MF2) of $B$. rapa, $B$. oleracea, and $B$. napus (AA and $\mathrm{CC}$ genomes) were counted. The gene loci with tandem repeats were calculated using one gene. The 458 core genes and 459 random genes of $A$. thaliana were downloaded from the CEGMA database (http://korflab.ucdavis.edu/Datasets/cegma); the homologous genes of 917 genes in $B$. rapa, $B$. oleracea and $B$. napus were searched in $B R A D$, and their retention rates were counted [62, 63]. The retention and loss of genes on the AA and $C C$ genome in $B$. napus during tetraploid were analyzed.

Motif analysis, signal peptide, and subcellular localization prediction in B. oleracea, B. rapa and B. napus

MEME was used to analyze the common conserved the short amino acid sequence of bHLH family members [64]. In this study, we searched 15 bHLH gene motifs. Then, the conserved motifs were integrated with phylogenetic trees by using Photoshop CS3. SignalP-4.1 (http://www.cbs.dtu.dk/services/SignalP/) was utilized to predict the signal peptide of the bHLH genes in B. oleracea, B. rapa and B. napus, all of which are set at default [65]. We can predict the position of signal peptide according to the values of $\mathrm{C}, \mathrm{S}$ and $\mathrm{Y}$ in the results. Each amino acid corresponds to one $S$ value, and the value of signal peptide region is higher; Each amino acid has a $C$ value, and the highest $C$ value is the shear site. Y-max value was used to predict the shear site, where $S$ value is the steep position and the site with high $C$ value.

In order to confirm the predicted results more reliable, three prediction methods were used to predict the subcellular localization of bHLH gene. They are WolfPsort (https:// wolfpsort.hgc.jp) [66], Plant-mPLoc (http://www.csbio.sjtu. edu.cn/bioinf/plant-multi/) [67] and ProtComp 9.0 (http:// linux1.softberry.com). If two or more of the predicted results are identical, it is regarded as the subcellular localization of the encoded protein. BrabHLH051, BrabHLH245, BolbHLH207 and BnabHLH024 were randomly selected for homeopathic expression in tobacco cells. The CDS sequences of these genes were amplified from B. rapa, B. oleracea and B. napus with a high-fidelity enzyme (Vazyme, China). The primers were seen in Table S11. We constructed the CDS sequences on the pFGC vector containing GPF fluorescence marker by homologous recombination. After that, the constructed vectors were injected into tobacco leaves, and the GFP fluorescence signal in tobacco leaf cells was observed by laser confocal microscope LSM780 (ZEISS, Germany) after $36 \mathrm{~h}$ of growth.
Plant material, hormone treatments and expression pattern analyses of bHLH genes in B. oleracea, $B$. rapa and $B$. napus

The plant materials (B. rapa accession Chiifu-401-42 and $B$. napus $\mathrm{cv}$. Zheyou 606) for expression patterns analyses were planted in the experimental farm of Zhejiang University. $B$. oleracea cv. Zhegan No. 1 was grown in the experimental farm of Zhejiang Academy of Agricultural Science. All plant materials were not deposited in a publicly available herbarium. Roots, stems, leaves, flowers, silique, big bud $(>2.0$ $\mathrm{mm})$, middle buds, and small bud $(<2.0 \mathrm{~mm})$ in the flowering period were sampled in liquid nitrogen and stored in a refrigerator at $-80^{\circ} \mathrm{C}$. The plant materials (B. rapa, B. oleracea and $B$. napus) for hormone treatments were planted in the glass greenhouse. The leaves of the three Brassica crops were treated with $\mathrm{ABA}$ and JA. When the plants grew five true leaves, the leaves were sprayed with $100 \mu \mathrm{M}$ ABA and $100 \mu \mathrm{M} \mathrm{JA}$, and samples were collected at $0 \mathrm{~h}, 0.5 \mathrm{~h}, 1 \mathrm{~h}$ and $2 \mathrm{~h}$ after treatments. All samples were frozen in liquid nitrogen and stored in a refrigerator at $-80^{\circ} \mathrm{C}$.

Total RNA was extracted from previous materials by using the TRIzol reagent (Invitrogen, USA) following the manufacturer's instructions. The first cDNA strand was generated following the manufacturer's protocol by using the Takara Reverse Transcription System (Japan). A total of 50 B. oleracea, 50 B. rapa, and $65 \mathrm{~B}$. napus bHLH genes were chosen to analyze the expression patterns, and these genes were orthologous genes. The primers used for qRT-PCR in $B$. oleracea, B. rapa and B. napus are shown in Table S12, S13, and S14, respectively. We selected 20 genes from each crop and analyzed their expression patterns after treatments. The primers were designed using Integrated DNA Technologies (https://sg.idtdna.com/pages) and the Primer (version 5.0) software. All cDNA samples were adjusted to a uniform concentration. SYBR Green Master Mix Reagent (TOYOBO, Japan) were used to do the Realtime fluorescent quantitative PCR. Three technical replicates for each sample were performed in a realtime PCR machine (Bio-Rad CFX Manager). To normalize the total amount of cDNA present in each reaction, we amplified the gene $U B C 10$ of $B$. rapa, GAPDH of $B$. oleracea and $25 S$ of $B$. napu as endogenous controls in calibrating the relative expression [68-70]. The $2^{-\triangle \Delta C T}$ method of the relative gene quantification recommended by Applied Biosystems (PE Applied Biosystems, USA) was used to calculate the expression level of different tissues [71]. For expression patterns analyses, we set the tissue with the lowest gene expression as the control, calibrated the expression value to 1 , and calculated the relative gene expression in other tissues. For the treatments, the samples collected at $0 \mathrm{~h}$ after treatments were set as control. The relative expression were taken as a logarithmic form of all genes and make a heat map with Helm software [72]. 


\section{Supplementary information}

Supplementary information accompanies this paper at https://doi.org/10. 1186/s12864-020-6572-6

Additional file 1: Figure S1. Phylogenetic tree of bHLH genes of $B$ oleracea, B. rapa, B. napus and A. thaliana. Branches of the bHLH genes in $A$. thaliana and $B$. rapa were labeled in red and green, respectively. The numbers on the branches indicate the bootstrap percentage values calculated from 1000 replicates.

Additional file 2: Figure S2. Phylogenetic tree of $B$. oleracea bHLH genes with domain sequences. The numbers on the branches indicate the bootstrap percentage values calculated from 1000 replicates.

Additional file 3: Figure S3. Phylogenetic tree of bHLH genes of AA genome of $B$. napus. The numbers on the branches indicate the bootstrap percentage values calculated from 1000 replicates.

Additional file 4: Figure S4. Phylogenetic tree of bHLH genes of CC genome of $B$. napus. The numbers on the branches indicate the bootstrap percentage values calculated from 1000 replicates.

Additional file 5: Figure S5. Phylogenetic tree of B. rapa bHLH genes with domain sequences. The numbers on the branches indicate the bootstrap percentage values calculated from 1000 replicates.

Additional file 6: Figure S6. Sequence logos of bHLH protein motifs of the three Brassica crops. Logos are a visualization tool for motifs. The height of a letter indicates its relative frequency at the given position.

Additional file 7: Figure S7. Conserved motifs analyses of bHLH genes in B. oleracea

Additional file 8: Figure S8. Conserved motifs analyses of bHLH genes in B. rapa

Additional file 9: Figure S9. Conserved motifs analyses of bHLH genes in B. napus. A: The conserved motifs of AA genome of B. napus; $B$ : The conserved motifs of CC genome of B. napus.

Additional file 10: Figure S10. Gene structure analyses of $\mathrm{bH} L \mathrm{H}$ genes in B. oleracea. Exons and introns are represented by boxes and lines, respectively.

Additional file 11: Figure S11. Gene structure analyses of bHLH genes in B. rapa. Exons and introns are represented by boxes and lines, respectively

Additional file 12: Figure S12. Gene structure analyses of bHLH genes in the $B$. napus. A: Exons- introns ananalyses of bHLH genes in AA genome of B. napus; B: Exons- introns ananalyses of bHLH genes in CC genome of $B$. napus. Exons and introns are represented by boxes and lines, respectively.

Additional file 13: Figure S13. Signal peptide prediction of BolbHLH128 (A), BrabHLH084 (B), and BrabHLH168 (C)

Additional file 14: Table S1. Identified bHLH genes in B. oleracea and their molecular characteristics

Additional file 15: Table S2. Identified bHLH genes in the AA genome of $B$. napus and their molecular characteristic

Additional file 16: Table S3. Identified bHLH genes in the CC genome of $B$. napus and their molecular characteristics

Additional file 17: Table S4. Identified bHLH genes in B. rapa with 2 newly identified BrabHLH genes and their molecular characteristics. The genes with yellow background were newly identified BrabHLH genes in this study, while the others were identified in a previous study (Song et al., 2014).

Additional file 18: Table S5. Nonsynonymous and synonymous substitution rates of orthologous bHLH genes between $B$. oleracea and $A$. thaliana

Additional file 19: Table S6. Nonsynonymous and synonymous substitution rates of orthologous bHLH genes between $A$. thaliana and $B$. rapa

Additional file 20: Table S7. Nonsynonymous and synonymous substitution rates of orthologous bHLH genes in $B$. napus
Additional file 21: Table S8. Subcellular location predictions of partial bHLH proteins

Additional file 22: Table S9. Original $C t$ values of selected genes of $B$. olerecea, B. rapa and B. napus

Additional file $\mathbf{2 3}$ Table S10. Relative expression levels of $20 \mathrm{Bo} / \mathrm{bHLH}$, $20 \mathrm{BrabHLH}$, and $20 \mathrm{BnabHLH}$ genes under ABA and JA treatments

Additional file 24: Table S11. Primer sequences of selected genes for subcellular localization analysis

Additional file 25 Table S12. Primers of selected bHLH genes for qRT$\mathrm{PCR}$ in B. oleraced

Additional file 26: Table S13. Primers of selected bHLH genes for qRTPCR in B. rapa

Additional file 27: Table S14. Primers of selected bHLH genes for qRTPCR in B. napus

\section{Abbreviations}

bHLH: Basic helix-loop-helix; CDS: Coding sequence; HEC: HECATE; IND: INDEHISCENT; MYA: Millions of years ago; ORF: Open reading frame; PMEl: Pectin methylesterase inhibitors; SDG: SET domain group; SPT: SPATULA; TCS: Two component signaling system; WGD: Whole genome duplication; WGT: Whole genome triplication

\section{Acknowledgements}

The authors gratefully acknowledge Dr. Longjiang Fan and Dr. Li Huang for their technological guidance and Dr. Zhenning Liu for stimulating discussions and critical reading of the manuscript. The original source of $B$ rapa seeds came from Dr. Xiaowu Wang of the Institute of vegetables and Flowers Chinese Academy of Agricultural Sciences. The seeds of B. napus were provided by Dr. Jianbin Li of Jiangsu Academy of Agricultural Sciences. The seeds of B. oleracea were provided by Dr. Xinmin Zhong. In this study, Dr. Xiaolin Yu undertook the formal identification of all plant material. We also thank them for offering the plant materials and seeds. If anyone require these seeds for study only, the corresponding author would like to send the seeds to them according to the relative agreement.

\section{Authors' contributions}

XLY and LMM proposed and supervised the research and gave final approval of the version to be published. LMM and YYG performed sequence analysis. YYG, KZ, and LJK performed qRT-PCR analysis. LMM, SBY, RRL, and KWL performed statistical analyses and interpreted the experimental results. LMM and XLY wrote the manuscript.

\section{Funding}

This work was partially supported by the National Key Research and Development Program of China (grant no. 2016YFD0100204-31), National Natural Science Foundation of China (grant nos. 31872110 and 31700272), the Breeding Project of the Sci-tech Foundation of Zhejiang Province (grant no. 2016C02051-6-1), the Project of SRTP in Zhejiang University (grant no. 2018R401080), the Project of Sci-tech Foundation of Jiangsu Province (grant no. BE2018310), and the Project of Application on Public Welfare Technology in Zhejiang Province (grant no. LGN18C150003). The funding agencies had no role in the design, analysis, and interpretation of the data or writing of the manuscript. All funders we mentioned provide financial support for our study.

\section{Availability of data and materials}

All sequences used in this study can be found in Brassica Database (http:// brassicadb.org/brad/) according to their gene ID numbers. In addition, the genome sequences of $B$. oleracea used for identifying the bHLH genes in this study were deposited in the DDBJ/EMBL/GenBank nucleotide core database under the accession code AOIX00000000; The genome sequences of $B$. rapa used for identifying the bHLH genes were deposited at DDBJ/ EMBL/GenBank under the accession AENI00000000; Sequence Read Archive accession numbers of $B$. napus sequencing data are ERP005275 and PRJEB6069, which been used to identify the bHLH genes in B. napus. The other data sets generated in this study are included within the article and supplementary files. All materials used or generated during the study are 
kept in our laboratory and are available from the corresponding author by reasonable request.

\section{Ethics approval and consent to participate}

Not applicable. This study has not directly involved humans, animals. We comply with the Convention on the Trade in Endangered Species of Wild Fauna and Flora.

\section{Consent for publication}

Not applicable.

\section{Competing interests}

All authors declare that they have no competing interests.

Received: 27 May 2019 Accepted: 10 February 2020

\section{Published online: 24 February 2020}

\section{References}

1. Liu S, Liu Y, Yang X, Tong C, Edwards D, Parkin IA, et al. The Brassica oleracea genome reveals the asymmetrical evolution of polyploid genomes. Nat Commun. 2014;5:3930.

2. William R, Atchley WT, Dress A. Positional dependence, cliques, and predictive motifs in the bHLH protein domain. J Mol Evol. 1999;48:501-16.

3. Li X, Duan X, Jiang H, Sun Y, Tang Y, Yuan Z, et al. Genome-wide analysis of basic helix-loop-helix transcription factor family in rice and Arabidopsis. Plant Physiol. 2006:141(4):1167-84.

4. Murre C, Mccaw PS, Baltimore D. A new DNA binding and dimerization motif in immunoglobulin enhancer binding, daughterless, MyoD, and myc proteins. Cell. 1989;56(5):777-83.

5. Heim MA, Jakoby M, Werber M, Martin C, Weisshaar B. Bailey PC. The basic helix-loop-helix transcription factor family in plants: A genome-wide study of protein structure and functional diversity. Mol Biol \& Evol 2003, 20(5): 735-747.

6. William R, Atchley WMF. A natural classification of the basic helix-loop-helix class of transcription factors. P Natl Acad Sci USA. 1997;94:5172-6.

7. Komatsu K, Maekawa M, Ujiie S, Satake Y, Furutani I, Okamoto H, et al. LAX and SPA: major regulators of shoot branching in rice. Proc Natl Acad Sci U S A. 2003;100(20):11765-70.

8. Seo JS, Joo J, Kim MJ, Kim YK, Nahm BH, Song Sl, et al. OsbHLH148, a basic helix-loop-helix protein, interacts with OsJAZ proteins in a jasmonate signaling pathway leading to drought tolerance in rice. Plant J. 2011;65(6): 907-21.

9. Kiribuchi K, Sugimori M, Takeda M, Otani T, Okada K, Onodera H, et al. RERJ1, a jasmonic acid-responsive gene from rice, encodes a basic helixloop-helix protein. Biochem Biophys Res Commun. 2004;325(3):857-63.

10. Kiribuchi K, Jikumaru Y, Kaku H, Minami E, Hasegawa M, Kodama O, et al. Involvement of the basic helix-loop-helix transcription factor RERJ1 in wounding and drought stress responses in rice plants. Biosci Biotechnol Biochem. 2005;69(5):1042-4.

11. Zhou J, Li F, Wang JL, Ma Y, Chong K. Xu YY. Basic helix-loop-helix transcription factor from wild rice $(\mathrm{OrbHLH2})$ improves tolerance to salt- and osmotic stress in Arabidopsis. J Plant Physiol. 2009;166(12):1296-306.

12. Wang $Y J$, Zhang $Z G$, He XJ, Zhou HL, Wen YX, Dai JX, et al. A rice transcription factor OsbHLH1 is involved in cold stress response. Theor Appl Genet. 2003;107(8):1402-9.

13. Marcus GB, Heisler AA, Bylstra YH, Walsh R, Smyth DR. SPATULA, a gene that controls development of carpel margin tissues in Arabidopsis, encodes a bHLH protein. Development. 2001;128:1089-98.

14. Girin T, Paicu T, Stephenson P, Fuentes S, Korner E, O'Brien M, et al. INDEHISCENT and SPATULA interact to specify carpel and valve margin tissue and thus promote seed dispersal in Arabidopsis. Plant Cell. 2011; 23(10):3641-53.

15. Gremski K, Ditta G. Yanofsky MF. The HECATE genes regulate female reproductive tract development in Arabidopsis thaliana. Development 2007, 134(20):3593-3601

16. Sweeney MT, Thomson MJ, Pfeil BE.McCouch S. Caught red-handed: RC encodes a basic helix-loop-helix protein conditioning red pericarp in rice. Plant Cell 2006, 18(2):283-294.

17. Li X, Chen L, Hong M, Zhang Y, Zu F, Wen J, et al. A large insertion in bHLH transcription factor BrTT8 resulting in yellow seed coat in Brassica rapa. PLoS One. 2012;7(9):e44145
18. Zhang C, Feng R, Ma R, Shen Z, Cai Z, Song Z, et al. Genome-wide analysis of basic helix-loop-helix superfamily members in peach. PLoS One. 2018; 13(4):e0195974

19. Zhao F, Li G, Hu P, Zhao X, Li L, Wei W, et al. Identification of basic helixloop-helix transcription factors reveals candidate genes involved in anthocyanin biosynthesis from the strawberry white-flesh mutant. Sci Rep. 2018;8(1):2721.

20. Wang R, Zhao P, Kong N, Lu R, Pei Y, Huang C, et al. Genome-wide identification and characterization of the potato bHLH transcription factor family. Genes. 2018;9:54

21. Wang P, Su L, Gao H, Jiang X, Wu X, Li Y, et al. Genome-wide characterization of bHLH genes in grape and analysis of their potential relevance to abiotic stress tolerance and secondary metabolite biosynthesis. Front Plant Sci. 2018;9:64

22. Gao C, Sun J, Wang C, Dong Y, Xiao S, Wang X, et al. Genome-wide analysis of basic helix-loop-helix gene family in peanut and assessment of its roles in pod development. PLoS One. 2017;12(7):e0181843.

23. Song XM, Huang ZN, Duan WK, Ren J, Liu TK, Li Y, et al. Genome-wide analysis of the bHLH transcription factor family in Chinese cabbage (Brassica rapa ssp. pekinensis). Mol Gen Genomics. 2014;289(1):77-91.

24. Koch MA, Haubold B, Mitchell-Olds T. Comparative evolutionary analysis of chalcone synthase and alcohol dehydrogenase loci in Arabidopsis, Arabis, and related genera (Brassicaceae). Mol Biol Evol. 2000;17(10):1483-98.

25. Wang XW, Wang HZ, Wang J, Sun RF, Wu J, Liu SY, et al. The genome of the mesopolyploid crop species Brassica rapa. Nat Genet. 2011;43(10):1035U1157.

26. Yang JH, Liu DY, Wang XW, Ji CM, Cheng F, Liu BN, et al. The genome sequence of allopolyploid Brassica juncea and analysis of differential homoeolog gene expression influencing selection. Nat Genet. 2016;48(10): $1225-32$

27. Liu Z, LV Y, Zhang M, Liu Y, Kong L, Zou M, et al. Identification, expression, and comparative genomic analysis of the IPT and CKX gene families in Chinese cabbage (Brassica rapa ssp pekinensis). BMC Genomics. 2013;14:594.

28. Miao LM, LV YX, Kong LJ, Chen QZ, Chen CQ, Li J, et al. Genome-wide identification, phylogeny, evolution, and expression patterns of MtN3/saliva/ SWEET genes and functional analysis of BCNS in Brassica rapa. BMC Genomics. 2018;19:174

29. Kong L, Zhao K, Gao YY, Miao LM, Chen CQ, Deng H, et al. Comparative analysis of cytokinin response factors in Brassica diploids and amphidiploids and insights into the evolution of Brassica species. BMC Genomics. 2018;19:728.

30. Bowers JE, Chapman BA, Rong JK, Paterson AH. Unravelling angiosperm genome evolution by phylogenetic analysis of chromosomal duplication events. Nature. 2003;422(6930):433-38.

31. Town CD, Cheung F, Maiti R, Crabtree J, Haas BJ, Wortman JR, et al. Comparative genomics of Brassica oleracea and Arabidopsis thaliana revea gene loss, fragmentation, and dispersal after polyploidy. Plant Cell. 2006; 18(6):1348-59.

32. Yang YW, Lai KN, Tai PY. Li WH. Rates of nucleotide substitution in angiosperm mitochondrial DNA sequences and dates of divergence between Brassica and other angiosperm lineages. J Mol Evol 1999, 48(5):597-604.

33. Mun JH, Kwon SJ, Yang TJ, Seol YJ, Jin M, Kim JA, et al. Genome-wide comparative analysis of the Brassica rapa gene space reveals genome shrinkage and differential loss of duplicated genes after whole genome triplication. Genome Biol. 2009;10(10):R111.

34. He YJ, Liu X, Ye L, Pan CT, Chen LF, Zou T, et al. Genome-wide identification and expression analysis of two-component system genes in tomato. Int J Mol Sci. 2016;17(8):1204

35. Liu TT, Yu H, Xiong XP, Yue XY, Yu YJ, Huang L, et al. Genome-wide identification, molecular evolution, and expression profiling analysis of pectin methylesterase inhibitor genes in Brassica campestris ssp chinensis. Int J Mol Sci. 2018;19(5):1338.

36. Dong H, Liu DD, Han TY, Zhao YX, Sun J, Lin S, et al. Diversification and evolution of the SDG gene family in Brassica rapa after the whole genome triplication. Sci Rep. 2015:5:16851.

37. Mao K, Dong QL, Li C, Liu CH. Ma FW. Genome wide identification and characterization of apple bHLH transcription factors and expression analysis in response to drought and salt stress. Front Plant Sci 2017, 8:480

38. Chen $\mathrm{HC}$, Cheng WH, Hong CY, Chang YS. Chang MC. The transcription factor OsbHLH035 mediates seed germination and enables seedling recovery from salt stress through $A B A$-dependent and $A B A$-independent pathways, respectively. Rice 2018, 11:50. 
39. Jackson S, Chen ZJ. Genomic and expression plasticity of polyploidy. Curr Opin Plant Biol. 2010;13(2):153-9.

40. Hufton AL, Panopoulou G. Polyploidy and genome restructuring: a variety of outcomes. Curr Opin Genet Dev. 2009;19(6):600-6.

41. Blanc $\mathrm{G}$, Wolfe $\mathrm{KH}$. Functional divergence of duplicated genes formed by polyploidy during Arabidopsis evolution. Plant Cell. 2004;16(7):1679-91.

42. Van de Peer $Y$, Maere $S$, Meyer A. The evolutionary significance of ancient genome duplications. Nat Rev Genet. 2009;10(10):725-32

43. Kim J, Lee J, Choi JP, Park I, Yang K, Kim MK, et al. Functional innovations of three chronological mesohexaploid Brassica rapa genomes. BMC Genomics. 2014;15:606.

44. Yang XM, Ren YL, Cai Y, Niu M, Feng ZM, Jing RN, et al. Overexpression of OsbHLH107, a member of the basic helix-loop-helix transcription factor family, enhances grain size in rice (Oryza sativa L.). Rice. 2018;11:41.

45. Lim SD, Yim WC, Liu DG, Hu RB, Yang XH. Cushman JC. A Vitis vinifera basic helix-loop-helix transcription factor enhances plant cell size, vegetative biomass and reproductive yield. Plant Biotechnol J. 2018;16(9):1595-615.

46. Castelain M, Le Hir R, Bellini C. The non-DNA-binding bHLH transcription factor PRE3/6HLH135/ATBS1/TMO7 is involved in the regulation of light signaling pathway in Arabidopsis. Physiol Plantarum. 2012;145(3):450-60.

47. Zhang HB, Bokowiec MT, Rushton PJ, Han SC. Timko MP. Tobacco transcription factors NtMYC2a and NtMYC2b form nuclear complexes with the NtJAZ1 repressor and regulate multiple jasmonate-inducible steps in nicotine biosynthesis. Mol Plant 2012, 5(1):73-84.

48. Yang YF, Zhang KK, Yang LY, Lv X, Wu Y, Liu HW, et al. Identification and characterization of MYC transcription factors in Taxus sp. Gene. 2018;675:1-8.

49. Geng JJ, Liu JH. The transcription factor CsbHLH18 of sweet orange functions in modulation of cold tolerance and homeostasis of reactive oxygen species by regulating the antioxidant gene. J Exp Bot. 2018;69(10): 2677-92.

50. Huang DQ, Dai WH. Molecular characterization of the basic helix-loop-helix $(b H L H)$ genes that are differentially expressed and induced by iron deficiency in Populus. Plant Cell Rep. 2015;34(7):1211-24.

51. Babitha KC, Vemanna RS, Nataraja KN, Udayakumar M. Overexpression of EcbHLH57 transcription factor from Eleusine coracana L. in tobacco confers tolerance to salt, oxidative and drought stress. PLoS One. 2015;10(9):e0137098.

52. Pires N, Dolan L. Origin and diversification of basic-helix-loop-helix proteins in plants. Mol Biol Evol. 2010;27(4):862-74.

53. Toledo-Ortiz G, Huq E, Quail PH. The Arabidopsis basic helix-loop-helix transcription factor family. Plant Cell. 2003;15(8):1749-70.

54. Chalhoub B. Early allopolyploid evolution in the post-Neolithic Brassica napus oilseed genome. Science. 2014;345(6202):1255

55. Gotz S, Garcia-Gomez JM, Terol J, Williams TD, Nagaraj SH, Nueda MJ, et al. High-throughput functional annotation and data mining with the Blast2GO suite. Nucleic Acids Res. 2008;36(10):3420-35.

56. Voorrips RE. MapChart: software for the graphical presentation of linkage maps and QTLs. J Hered. 2002;93(1):77-8.

57. Thompson JD, Higgins DG, Gibson TJ. Clustal-W: improving the sensitivity of progressive multiple sequence alignment through sequence weighting, position-specific gap penalties and weight matrix choice. Nucleic Acids Res. 1994;22(22):4673-80.

58. Saitou N, Nei M. The neighbor-joining method -a new method for reconstructing phylogenetic trees. Mol Biol Evol. 1987;4(4):406-25.

59. Larkin MA, Blackshields G, Brown NP, Chenna R, McGettigan PA, McWilliam H, et al. Clustal W and clustal X version 2.0. Bioinformatics. 2007;23(21):2947-8.

60. Wang DP, Wan HL, Zhang S, Yu J. Y-MYN: a new algorithm for estimating Ka and Ks with consideration of variable substitution rates. Biol Direct. 2009:4: 20.

61. Krzywinski M, Schein J, Birol I, Connors J, Gascoyne R, Horsman D, et al. Circos: an information aesthetic for comparative genomics. Genome Res. 2009;19(9):1639-45.

62. Parra G, Bradnam K, Korf I. CEGMA: a pipeline to accurately annotate core genes in eukaryotic genornes. Bioinformatics. 2007;23(9):1061-7.

63. Cheng F, Wu J, Fang L. Wang XW. Syntenic gene analysis between Brassica rapa and other Brassicaceae species. Front Plant Sci 2012, 3:198.

64. Bailey TL, Boden M, Buske FA, Frith M, Grant CE, Clementi L, et al. MEME SUITE: tools for motif discovery and searching. Nucleic Acids Res. 2009;37: W202-8.

65. Petersen TN, Brunak S, von Heijne G. Nielsen H. SignalP 4.0: discriminating signal peptides from transmembrane regions. Nat Methods. 2011;8(10):785-6.
66. Horton P, Park KJ, Obayashi T, Fujita N, Harada H, Adams-Collier CJ, et al. WOLF PSORT: protein localization predictor. Nucleic Acids Res. 2007;35: W585-7.

67. Chou KC, Shen HB. Plant-mPLoc: a top-down strategy to augment the power for predicting plant protein subcellular localization. PLoS One. 2010;5: e11335.

68. Czechowski T, Stitt M, Altmann T, Udvardi MK, Scheible WR. Genome-wide identification and testing of superior reference genes for transcript normalization in Arabidopsis. Plant Physiol. 2005;139(1):5-17.

69. Broekgaarden C, Poelman EH, Steenhuis G, Voorrips RE, Dicke M, Vosman B. Responses of Brassica oleracea cultivars to infestation by the aphid Brevicoryne brassicae: an ecological and molecular approach. Plant Cell Environ. 2008;31(11):1592-605.

70. Shopan J, Mou HP, Zhang LL, Zhang CT, Ma WW, Walsh JA, et al. Eukaryotic translation initiation factor 2B-beta (elF2BB), a new class of plant virus resistance gene. Plant J. 2017;90(5):929-40.

71. Livak KJ, Schmittgen TD. Analysis of relative gene expression data using real-time quantitative PCR and the $2^{-\Delta \Delta C T}$ method. Methods. 2001;25(4): 402-8.

72. Deng WK, Wang YB, Liu ZX, Cheng H. Xue Y. Heml: A toolkit for illustrating heatmaps. PLoS One 2014, 9(11):e111988.

\section{Publisher's Note}

Springer Nature remains neutral with regard to jurisdictional claims in published maps and institutional affiliations.
Ready to submit your research? Choose BMC and benefit from:

- fast, convenient online submission

- thorough peer review by experienced researchers in your field

- rapid publication on acceptance

- support for research data, including large and complex data types

- gold Open Access which fosters wider collaboration and increased citations

- maximum visibility for your research: over 100M website views per year

At BMC, research is always in progress.

Learn more biomedcentral.com/submissions 